\title{
DESARROLLO DE COMPETENCIAS SOCIALMENTE RESPONSABLES EN LOS ALUMNOS DE BACHILLERATO DE EXCELENCIA MEDIANTE METODOLOGÍA DE CASCADA
}

The development of social responsibility competences in higher secondary school through methodology of cascade

Desenvolvimento de competências socialmente responsáveis em alunos do ensino médio através da metodologia de cascata

Financiación: Plan de Apoyo a Grupos de Innovación de la ULE (GID40).

\section{Daniel Alonso-Martínez (1)}

Beatriz Jiménez-Parra (2)

Mariano Nieto (3)

(1) Universidad de León, España. Teléfono: +34 987293499. Correo electrónico: dalom@unileon.es.

(2) Universidad de León, España. Teléfono: +34 987291982. Correo electrónico: beatriz.jimenez@unileon.es.

(3) Universidad de León, España. Teléfono: +34 987291876. Correo electrónico: mariano.nieto@unileon.es

\begin{abstract}
Resumen
El principal objetivo de la iniciativa es ofrecer un modelo educativo integrador que fomente la generación de ideas socialmente innovadoras y responsables. Al mismo tiempo, se busca trabajar las competencias generales para favorecer la interacción e integración de los estudiantes. Para ello, se ha utilizado la metodología de cascada. Concretamente, el instrumento utilizado es el poster. Los participantes de la iniciativa son 20 estudiantes de Bachillerato de la Excelencia y 4 del Grado en Administración y Dirección de Empresas de la Universidad de León. Los resultados alcanzados han sido cuatro ideas de negocio centradas en resolver problemas medioambientales a través de nuevas prácticas de gestión empresarial, y dos ideas socialmente innovadoras para Desarrollo de competencias socialmente responsables en los alumnos de bachillerato de excelencia mediante metodología de cascada


mejorar la atención, cuidado y la satisfacción de personas discapacitadas. Así, la iniciativa desarrollada ha permitido mejorar competencias académico-generales de los estudiantes, y al mismo tiempo les ha proporcionado una visión más cercana de la realidad empresarial y social.

Palabras clave: Creatividad; Innovación; Responsabilidad Social Corporativa; Metodología de Cascada

\begin{abstract}
The main objective of this initiative is to provide an integrative education model in order to foster the generation of social innovative and responsible ideas. Furthermore, the present initiative aims to develop the generic skills to stimulate interaction and integration among students. In this sense, the cascade methodology was the method and, in particular, poster was the specific instrument used. Two groups of students participated in this initiative: 20 students from secondary school (Higher secondary school) and 4 students of the Degree of Business Administration from the Universidad de León. As main results it can be highlighted (i) the generation of four business ideas focusing in solving some environmentally problems through the application of new practices of business management and (ii) the generation of two social innovative ideas to improve the attention, care and satisfaction of the disability people. Thus, the developed initiative has allowed students to improve their academic-generic skills and to obtain a better and closer point of view of the business and social reality.
\end{abstract}

Keywords: Creativity; Innovation; Corporate Social Responsability; Cascade Methodology

\title{
Resumo
}

O principal objetivo da iniciativa é oferecer um modelo educativo integrador que fomente a geração de idéias socialmente inovadoras e responsáveis. Ao mesmo tempo, se busca trabalhar as competências gerais para favorecer a interação e a integração dos estudantes. Para isso, usei a metodologia de cascada. Concretamente, um pôster é o instrumento utilizado. Os participantes da iniciativa são 20 estudantes de Bachillerato da Excelência e 4 de Grado em Administração da Universidad de León. Os resultados alcançados têm sido quatro idéias de negócio centradas em resolver problemas ambientais através de novas práticas de gestão empresarial, e duas idéias socialmente Desarrollo de competencias socialmente responsables en los alumnos de bachillerato de excelencia mediante metodología de cascada 
inovadoras para melhorar a atenção, cuidado e satisfação de pessoas com deficiência. Assim, a iniciativa desenvolveu-se para melhorar as competências académico-gerais dos estudantes, e ao mesmo tempo os têm uma visão mais cercana da realidade empresarial e social.

Palavras-chave: Criatividade; inovação; Responsabilidade Social Corporativa; Metodologia em cascata

\section{Introducción}

Existe una necesidad de ofrecer modelos educativos que fomenten la creatividad y la innovación de los alumnos desde una perspectiva socialmente responsable (Bhattacharya et al., 2008; Loveless et al., 2006). Las empresas demandan cada vez más modelos de negocio en los que la Responsabilidad Social Corporativa sea parte fundamental de su estructura organizativa. Además, los últimos estudios ponen de manifiesto la necesidad de trabajar las competencias interpersonales que favorezcan la integración y la interacción entre estudiantes (Rodríguez \& Vieira, 2009). Al mismo tiempo, trabajos previos señalan la importancia de trabajar las competencias académicas generales: instrumentales y sistémicas (Escalona \& Loscertales, 2005; Parvú et al., 2014). De esta forma, la iniciativa desarrollada y centrada en el desarrollo de ideas de negocios socialmente responsables busca fomentar la creatividad y la innovación entre los estudiantes. Por otra parte, el hecho de que en la iniciativa participen estudiantes de Bachillerato y de Universidad hace especialmente interesante el trabajo de las competencias interpersonales.

\section{Contextualización}

La experiencia docente mencionada se ha desarrollado dentro del programa del Bachillerato de la Excelencia. En concreto, la presente iniciativa ha contado con la participación activa de docentes y estudiantes de dos organismos, un instituto de educación secundaria español y la Universidad de León. Las prácticas docentes se han desarrollado durante dos cursos académicos: 2015-2016 y 2016-2017, respectivamente. Esta práctica docente tiene como fin último la integración social y cultural de los estudiantes, así como el fomento de la gestión empresarial socialmente responsable. 
Asimismo, la iniciativa pretende trabajar las competencias instrumentales, interpersonales y sistémicas, tanto en estudiantes de Bachillerato como de Universidad.

\section{Diseño y desarrollo}

La presente iniciativa se centra en el fomento de la gestión empresarial socialmente responsable entre estudiantes de Bachillerato de Excelencia y estudiantes de Grado de una Universidad española. Durante la iniciativa, se crearon grupos de trabajo, formados, cada uno de ellos, por cinco estudiantes de Bachillerato y un estudiante de Grado. En primer lugar, se les proporcionó una breve formación acerca de la gestión social, y posteriormente se les planteó un reto (Tabla 1). En particular, este reto consistía en la creación de una idea socialmente innovadora centrada en la gestión socialmente responsable. Para ello, cada uno de los grupos debía elaborar un Poster que recogiera los principales aspectos de la idea (localización, público objetivo, ingresos, personal, etc.). En todos los casos, se les propuso un marco común en el que desarrollar sus ideas de negocio. En concreto, se establecieron dos requisitos: las ideas debían estar localizadas en una región concreta de España y el objetivo final de las mismas debía ser la generación de valor social para el territorio. Durante dicho reto, los estudiantes de Grado ejercían como “docentes improvisados”, asimismo los profesores implicados (2 de universidad y 1 de instituto) ejercían como supervisores y guiaban a los estudiantes para trabajar las competencias mencionadas.

La iniciativa desarrollada se enfocaba al trabajo de las competencias interpersonales, ya que permitían a los estudiantes involucrados mejorar su integración con otros estudiantes. De esta forma, los estudiantes entrenaban sus capacidades para saber cómo trabajar en equipo y comunicarse de forma efectiva con los demás. Es decir, para poder avanzar de forma satisfactoria en sus respectivas ideas de negocio, dichos estudiantes interactuaban, tomaban decisiones y se enfrentaban a situaciones diversas. De forma más concreta, la metodología predominante en esta práctica docente es la metodología de cascada. Mediante ésta, los estudiantes experimentan un cambio de rol, convirtiéndose en docentes. Así, los estudiantes universitarios ponen en valor los conocimientos y capacidades adquiridas durante sus años en la Universidad, al ejercer como docentes durante el desarrollo de la actividad. Por su parte, los estudiantes de Bachillerato interactúan y se integran en equipos de trabajo con "modelos de referencia" universitarios, lo que facilita su comprensión del mundo universitario y les permite Desarrollo de competencias socialmente responsables en los alumnos de bachillerato de excelencia 
trabajar de forma práctica y dinámica en actividades empresariales. Este tipo de metodología, permite explotar las sinergias que surgen entre los estudiantes y mejorar su nivel de creatividad e innovación. A través de los posters, los estudiantes trabajan las competencias instrumentales, y aplican los contenidos teórico-prácticos adquiridos en sus centros de origen. Al finalizar la iniciativa, los estudiantes poseen una visión de conjunto de lo que supone una idea empresarial socialmente innovadora. Además, los estudiantes presentan de forma oral sus ideas socialmente responsables, trabajando así las competencias sistémicas.

Tabla 1.

Fases de la iniciativa

\begin{tabular}{ll}
\hline Fase & Duración \\
\hline Contenidos teórico-prácticos impartidos & 30 minutos \\
Trabajo en grupo de las ideas socialmente innovadoras & 60 minutos \\
Presentación pública de las ideas & 30 minutos \\
Total & $\mathbf{2}$ horas \\
\hline
\end{tabular}

\section{Evaluación}

Los resultados obtenidos durante las dos ediciones de esta iniciativa ponen de manifiesto el compromiso de los estudiantes participantes con las iniciativas socialmente innovadoras y responsables. Las ideas de negocio desarrolladas durante el reto estaban bien argumentadas, con planteamientos cercanos a la realidad, que resuelven problemas hasta el momento poco abordados. En concreto, los proyectos desarrollados durante el curso académico 2015/16 estaban centrados en aspectos medioambientales, mientras que los proyectos desarrollados en la última edición se centraban en colectivos discapacitados (discapacitados con dificultades motoras y psicomotrices).

Al finalizar la actividad, todos los participantes fueron encuestados a través de una página web creada a tal efecto (http://conferenciaiesjde.weebly.com/bachilleratoexcelencia-juan-del-enzina.html). En todos los casos, los estudiantes a través de sus encuestas, señalaban como su percepción sobre la Responsabilidad Social Corporativa había cambiado después de la iniciativa. Además, ambos grupos valoraban especialmente la participación de estudiantes universitarios en la actividad. 


\section{Conclusiones}

Tal y como se ha comentado, los estudiantes valoraron muy positivamente la iniciativa desarrollada, haciendo especial énfasis a la involucración de estudiantes universitarios. Cabe señalar que uno de los objetivos de la iniciativa el trabajo de las competencias interpersonales. En este sentido, la interacción y cooperación inter-grupos desarrollada en el aula, permitió a los estudiantes desarrollar una mayor capacidad crítica y autocrítica, y mejorar su capacidad para apreciar la diversidad y la multiculturalidad, lo que es especialmente relevante en el ámbito empresarial. Además, la temática social del reto mejoró el compromiso y la concienciación de los estudiantes con los problemas sociales del mundo.

\section{Referencias}

Bhattacharya, C. B., Sen, S., \& Korschun, D. (2008). Using Corporate Social Responsibility to Win the War for Talent. MIT Sloan Management Review, 49(2), $1-10$.

Escalona, A. I., \& Loscertales, B. (2005). Actividades para la enseñanza y el aprendizaje de competencias genéricas en el marco del Espacio Europeo de Educación Superior. Zaragoza: Prensas Universitarias de Zaragoza.

Loveless, A., Burton, J., \& Turvey, K. (2006). Developing conceptual frameworks for creativity, ICT and teacher education. Thinking Skills and Creativity, 1(1), 3-13.

Parvu, I.; Ipate, D. M., \& Mitran, P. C. (2014). Identification of employability skills e starting point for the curriculum design process. Economics, Management, and Financial Markets, 9(1), 237-246.

Rodríguez, A., \& Vieira, M. (2009). La formación en competencias en la universidad: un estudio empírico sobre su tipología. Revista De Investigación Educativa, 27(1), 27-47.

Desarrollo de competencias socialmente responsables en los alumnos de bachillerato de excelencia mediante metodología de cascada 